\title{
Prevalence of Hypertension in the Middle Belt of Ghana: A Community-Based Screening Study
}

\author{
David Kwame Dosoo ${ }^{D},{ }^{1}$ Solomon Nyame, ${ }^{1}$ Yeetey Enuameh, ${ }^{2}$ Harold Ayetey, ${ }^{3,4}$ \\ Harry Danwonno, ${ }^{1}$ Mieks Twumasi, ${ }^{1}$ Cephas Tabiri, ${ }^{1}$ Stephaney Gyaase, ${ }^{1}$ \\ Gregory Y. H. Lip, ${ }^{5,6}$ Seth Owusu-Agyei, ${ }^{1,7}$ and Kwaku Poku Asante ${ }^{1}{ }^{1}$ \\ ${ }^{1}$ Kintampo Health Research Centre, Ghana Health Service, Kintampo, Ghana \\ ${ }^{2}$ Kwame Nkrumah University of Science and Technology, Kumasi, Ghana \\ ${ }^{3}$ University of Cape Coast School of Medical Sciences, Cape Coast Ghana, Ghana \\ ${ }^{4}$ Royal Brompton and Harefield NHS Trust, London, UK \\ ${ }^{5}$ Liverpool Centre for Cardiovascular Science, University of Liverpool and Liverpool Heart \& Chest Hospital, Liverpool, UK \\ ${ }^{6}$ Aalborg Thrombosis Research Unit, Department of Clinical Medicine, Aalborg University, Aalborg, Denmark \\ ${ }^{7}$ Institute of Health Research, University of Health and Allied Sciences, Ho, Ghana
}

Correspondence should be addressed to Kwaku Poku Asante; kwakupoku.asante@kintampo-hrc.org

Received 18 April 2019; Revised 6 August 2019; Accepted 17 September 2019; Published 7 October 2019

Academic Editor: Franco Veglio

Copyright (C) 2019 David Kwame Dosoo et al. This is an open access article distributed under the Creative Commons Attribution License, which permits unrestricted use, distribution, and reproduction in any medium, provided the original work is properly cited.

Objective. Prevalence of hypertension is on the rise and can be attributed to aging populations and changing behavioral or lifestyle risk factors. The objectives of this study were to determine the prevalence, awareness, treatment, control, and risk factors of hypertension in the middle part of Ghana. Methods. A total of 2,555 participants aged $\geq 18$ years (mean age of 43 years; $60.5 \%$ female) were enrolled using a two-stage sampling method. The World Health Organization STEPwise approach to chronic disease risk factor Surveillance-Instrument v2.1 was used for data collection. Blood pressure and anthropometric measurements were assessed. Blood glucose and lipids were also measured using blood samples collected after an overnight fast. Results. Prevalence of hypertension was $28.1 \%$ (95\% CI: 26.3\%-29.8\%). Less than half, i.e., $45.9 \%$ (95\% CI: $42.2 \%-49.6 \%$ ), of the respondents were aware of their hypertensive status. Of those aware and had sought medical treatment, $41.3 \%$ (95\% CI: 36.1-46.8) had their hypertension controlled. Risk factors associated with being hypertensive were current $(p=0.053)$ and past tobacco usage $(p<0.001)$, prediabetes $(p=0.042)$, high body mass index $(p<0.001)$, hyperglycaemia $(p=0.083)$, and hypercholesterolaemia $(p=0.010)$. Doing vigorous work and being active in sports were less associated with being hypertensive $(p<0.001)$. Conclusion. Our study showed that close to one-quarter of adults who were involved in the survey in the middle belt of Ghana were hypertensive with less than half being aware of their hypertensive status; nearly half of those on treatment had controlled hypertension. Healthcare systems need adequate resources that enable them to screen, educate, and refer identified hypertensive patients for appropriate management to prevent or minimize the development of hypertension-related complications.

\section{Introduction}

Hypertension is a major public health challenge, affecting populations from both economically developed and developing countries globally $[1,2]$. The disease remains a major risk factor for development of cardiovascular disease (CVD), which was responsible for 17.7 million deaths worldwide in 2015 (i.e., $45 \%$ of deaths due to noncommunicable diseases) [3]. The highest prevalence of hypertension in the world occurs in sub-Saharan Africa [4]. Many patients with hypertension are asymptomatic and predisposed to fatal cardiovascular events such as strokes and myocardial infarctions; hence, the the disease is described as a "silent killer" [5]. Depending on the aetiology, 
hypertension could be classified as primary (affecting 90$95 \%$ of patients) or secondary (resulting from an underlying condition) [5].

Although the exact cause of primary hypertension is unknown, there are several nonmodifiable and modifiable risk factors that have been linked to the condition. Nonmodifiable risk factors for hypertension include age, sex, race, family history, and genetic composition, whereas modifiable risk factors include obesity, excessive salt intake, inactivity or lack of exercise, high fat diet, tobacco use, and alcohol consumption [5-7]. According to the 2014 Ghana Demographic and Health Survey (GDHS), the overall prevalence of hypertension among persons aged 15-49 years was $13.0 \%$, with nearly half of them being aware of their hypertensive status [8]. A systematic review and metaanalysis by Bosu also reported a prevalence of adult hypertension between $19 \%$ and $48 \%$ [9].

Although the prevalence of hypertension was determined as part of the GDHS in 2014, this survey did not include biochemical measurements as outlined in the WHO STEPwise approach to chronic disease risk factor Surveillance-Instrument v2 due in part to cost considerations. A well-structured epidemiological survey to determine the prevalence, management, and risk factors including biochemical risk are vital to compliment that of the GDHS for the implementation and evaluation of the impact of interventions to control hypertension. A multiphased Kintampo Noncommunicable diseases (NCD) Initiative (KNI) programme incorporating the WHO STEPwise approach was established at the Kintampo Health Research Centre (KHRC) in 2015 to build capacity for NCD epidemiologic studies that would lead to interventional studies. This community-based epidemiological screening survey that establishes the burden of hypertension in the middle belt of Ghana initiated the programme.

\section{Methods}

2.1. Study Design and Setting. A community-based crosssectional study was conducted in two contiguous districts (Kintampo North Municipality and Kintampo South District) in the middle part of Ghana between October 2015 and December 2016. The study area, which lies in the geographical center of Ghana, has a total resident population of about 129,000 of which about two-thirds is rural [10]. There were two government hospitals, two private hospitals, four health centers, one private clinic, 25 community-based health planning and services (CHPS) areas, and two maternity homes that serve the population. The KHRC maintains a Health and Demographic Surveillance System (HDSS), which collects core information such as births, deaths, and migrations (in and out). Houses within the study area have been numbered and digitized, facilitating tracing of potential study participants in their homes [10].

Five days of training was organized for the clinical, laboratory, field, and data teams involved in the study covering implementation of the study protocol, specifically interviewing techniques, informed consenting process, anthropometric measurements, completion of the study questionnaire, and referral systems. Theoretical presentations and role play were employed during training sessions. Study tools were piloted within the community prior to commencement of the study.

2.2. Ethical Considerations. Ethical approval was obtained from the Kintampo Health Research Centre Institutional Ethics Committee (KHRCIEC/2014-25). Individual written informed consent was obtained from each participant before study procedures were carried out.

2.3. Sample Size. Based on an overall hypertension prevalence of $13.0 \%$ in the Ghanaian population status [8], a prevalence of $9.5 \%$ was used for the study area. Assuming a 0.05 type I error rate and a 0.011 margin of error, a minimum sample of 2,482 was estimated. Assuming a 10\% nonresponse rate, a sample size of 2,730 was used. The sample size calculation was based on the following formula:

$$
n=Z_{1-(\alpha / 2)}^{2} \frac{P(1-P)}{d^{2}},
$$

where $n$ =sample size, $Z_{1-(\alpha / 2)}=$ type $\mathrm{I}$ error rate $(0.05=1.96), P=$ assumed hypertension prevalence $(13.0 \%)$, and $d=$ margin of error $(0.011)$.

2.4. Selection of Study Communities and Participants. The sampling frame was the database of the Kintmapo Health and Demographic Surveillance System (KHDSS). A twostage sampling procedure was used in selecting the study population, ensuring that they were representative of the KHDSS population. In the first stage, 34 of the 164 communities in the KHDSS area were randomly selected using computer-generated random numbers and ensuring that the entire target population was covered. This was followed by visits to the leaders of the selected communities to explain the study to them and to seek their permission for participation of members' of their communities. The second stage involved stratification by gender and ascending age, of all individuals of 18 years and older in the selected communities. Potential study participants were then selected at random from the identified communities using a systematic sampling technique, which involved the selection of an "indexed" individual from the database of each selected community followed by the selection of every tenth individual. Listings of potential study participants were used by our study field workers who met the participants at home in the communities to explain the study to them and also invite them to a central location within the community for them to go through study procedures. Inclusion criteria were adult male and female subjects aged $\geq 18$ years who have been resident in the study area for at least 6 months and were willing to be part of the study.

2.5. Study Procedures. Following informed consent, questionnaires were administered to obtain demographic data, information on tobacco use, alcohol consumption, diet, physical activity, history of raised blood pressure, and 
diabetes, using the World Health Organization (WHO) STEPwise approach to chronic disease risk factor Surveillance-Instrument v2.1 [11]. All administered questionnaires were checked for completeness in the field and before data entry.

2.6. Blood Pressure Measurement. Three sedentary blood pressure (BP) measurements were taken at 3-minute intervals by a physician assistant using an Omron digital BP monitor (Omron Healthcare Co. Kyoto, Japan) with a cuff of appropriate width after the participant had sat for at least 15 minutes and relaxed.

2.7. Body Weight and Height Measurement. Two trained field staff measured and verified the body weight and height with the participant standing motionless in an erect position against the vertical surface of a standard height meter attached to a calibrated weighing scale. Participants had on minimum clothing and no footwear while their body weight and height were being measured.

2.8. Laboratory Measurements. Overnight fasting blood samples $(3 \mathrm{ml})$ were obtained by sterile venipuncture from each participant and dispensed into $1 \mathrm{ml}$ fluoride-EDTA and $2 \mathrm{ml}$ lithium heparin tubes for the estimation of glucose and lipids, respectively. Plasma was separated after centrifugation of blood at $1000 \mathrm{~g}$ for $10 \mathrm{~min}$. Glucose, cholesterol, and triglyceride levels were determined the same day using a Flexor $E$ automated Clinical Chemistry analyzer (Vital Scientific, The Netherlands) according to approved standard operating procedures. Samples that were to be analyzed later were kept frozen at $-20^{\circ} \mathrm{C}$ or below and thawed prior to analysis. Freeze-thaw cycles were avoided.

2.9. Outcome Definitions. We used definitions by the Seventh Report of the Joint National Committee on the Prevention, Detection, Evaluation and Treatment of High Blood Pressure [12]. Hypertension was defined as a mean systolic blood pressure (SBP) of $\geq 140 \mathrm{mmHg}$ and/or a mean DBP of $\geq 90 \mathrm{mmHg}$ or a respondent self-report of clinician diagnosis of hypertension or self-report of medication to lower blood pressure. Prehypertension was also defined as SBP of $120-139 \mathrm{mmHg}$ and DBP of $80-89 \mathrm{mmHg}$; stage 1 hypertension as SBP of $140-159 \mathrm{mmHg}$ and/or DBP 90$99 \mathrm{mmHg}$; and stage 2 hypertension as SBP of $\geq 160 \mathrm{mmHg}$ and/or DBP $\geq 100 \mathrm{mmHg}$. Awareness was defined as selfreport of a diagnosis of hypertension by a health worker. Control was defined as self-reported taking of medication to lower blood pressure with SBP of $<140 \mathrm{mmHg}$ and DBP of $<90 \mathrm{mmHg}$. Aware yet not on medication was defined as self-reported diagnosis by a health worker yet not taking medication to lower blood pressure. Diabetes mellitus was defined as fasting blood glucose level of $\geq 7.0 \mathrm{mmol} / \mathrm{L}$ or reported use of blood glucose lowering medication, whereas hypercholesterolemia and hypertriglyceridemia were defined as total cholesterol and triglyceride levels of $\geq 5.0 \mathrm{mmol} / \mathrm{L}$ and $\geq 2.2 \mathrm{mmol} / \mathrm{L}$, respectively.
Participants found to be hypertensive or having any other medical condition were counseled by a study clinician and referred to a health facility for treatment. All participants with a measured SBP of $\geq 140 \mathrm{mmHg}$ and/or DBP of $\geq 90 \mathrm{mmHg}$ were referred to the Municipal/District hospital or to their clinicians for appropriate management.

2.10. Data Management, Statistical Methods, and Analysis. Data were double entered, verified using (C Sharp) Visual Studio 2013 as front end and SQL server as back end prior to analysis. Means of second and third blood pressure measurements were used for the analysis. Measurement of household socioeconomic status was based on respondent's household assets. The assets were scored, and eigenvalues were computed using principal component analysis. By this method, the weighted assets were grouped into various components. The first component was used to generate the wealth quintiles. Variables were summarized using descriptive statistics. Chi-square test was used to find the relationship between being hypertensive and known risk factors. The analysis was done in Stata version 14.0 (StataCorp, USA).

\section{Results}

A total of 2,555 participants ( $60.5 \%$ female) from the HDSS area were enrolled into the survey following consenting (Table 1). Participants' enrolment was proportional to the population structure. For the risk set, those aged 18-39 years were in the majority (45.3\%), followed by those aged $40-$ 59 years $(35.7 \%)$. Approximately $41 \%$ of participants had no education, and $15.6 \%$ had a postsecondary educational status (e.g., training colleges and polytechnics) as their highest educational level. Majority (29\%) of the participants were from households with least poor socioeconomic status.

3.1. Prevalence of Hypertension. The overall prevalence of hypertension in the surveyed population was $28.1 \%$ (95\% CI: $26.3-29.8 \%$; 717/2555), and this increased with age. Agespecific prevalence of hypertension for participants aged 18 - 39 years, $40-59$ years, and $\geq 60$ years were $12.9 \%$ (95\% CI: $11.1-15.0 \%), 33.8 \%$ (95\% CI: $30.8-37.0 \%)$, and $53.3 \%$ (95\% CI: $48.8-57.7 \%)$, respectively.

Prevalence of the different types of raised blood pressure, i.e., prehypertension, systolic hypertension, diastolic hypertension, stage 1 hypertension, and stage 2 hypertension were $42.5 \%$ (95\% CI: $40.6 \%-44.5 \%$ ), $8.5 \%$ (95\% CI: $7.5 \%-$ 9.7\%), 3.2\% (95\% CI: $2.6 \%-3.9 \%$ ), $15.8 \%$ (95\% CI: $14.4 \%-$ $17.2 \%)$, and $8.9 \%$ (95\% CI: $7.9 \%-10.1 \%)$, respectively (Table 2).

Diastolic hypertension was the least common type of hypertension in the population but was most prevalent within 40-59 years. With the exception of diastolic hypertension, participants aged $\geq 60$ years generally had the most prevalent proportion for all the various types of hypertension. Close to a fifth (19\%) of those aged $\geq 60$ years had stage 2 hypertension (Table 2). 
Table 1: Distribution of respondents' basic demographic characteristics.

\begin{tabular}{|c|c|c|c|c|}
\hline \multirow[b]{2}{*}{ Demographic characteristics } & \multicolumn{3}{|c|}{ Respondent's age group } & \multirow{2}{*}{$\begin{array}{c}\text { Overall total }(2,555) \\
n(\%)\end{array}$} \\
\hline & $\begin{array}{c}18-39 \text { years } \\
N=1,158 \\
n(\%)\end{array}$ & $\begin{array}{c}40-59 \text { years } \\
N=913 \\
n(\%)\end{array}$ & $\begin{array}{c}60 \text { and above years } \\
N=484 \\
n(\%)\end{array}$ & \\
\hline \multicolumn{5}{|l|}{ Gender } \\
\hline Male & $441(38.1)$ & $353(38.7)$ & $215(44.4)$ & $1,009(39.5)$ \\
\hline Female & $717(61.9)$ & $560(61.3)$ & $269(55.6)$ & $1,546(60.5)$ \\
\hline \multicolumn{5}{|l|}{ Residence type } \\
\hline Urban & $467(40.3)$ & $293(32.1)$ & $151(31.2)$ & $911(35.7)$ \\
\hline Rural & $691(59.7)$ & $620(67.9)$ & $333(68.8)$ & $1,644(64.3)$ \\
\hline \multicolumn{5}{|l|}{ Education status } \\
\hline None & $246(21.2)$ & $455(49.8)$ & $342(70.7)$ & $1,043(40.8)$ \\
\hline Primary & $194(16.7)$ & $131(14.3)$ & $42(8.7)$ & $367(14.4)$ \\
\hline Secondary & $436(37.6)$ & $238(26.1)$ & $73(15.1)$ & $747(29.2)$ \\
\hline Postsecondary & $282(24.3)$ & $89(9.7)$ & $27(5.6)$ & $398(15.6)$ \\
\hline \multicolumn{5}{|l|}{ Registered with NHIS ${ }^{1}$} \\
\hline Yes & $484(41.8)$ & $464(50.8)$ & $290(59.9)$ & $1,238(48.4)$ \\
\hline No & $674(58.2)$ & $449(49.2)$ & $194(40.1)$ & $1,317(51.6)$ \\
\hline \multicolumn{5}{|l|}{$\mathrm{HH}_{\mathrm{SES}}{ }^{2}$} \\
\hline Least poor & $330(28.5)$ & $272(29.8)$ & $144(29.7)$ & $746(29.2)$ \\
\hline Less poor & $287(24.8)$ & $215(23.5)$ & $122(25.2)$ & $624(24.4)$ \\
\hline Poor & $229(19.8)$ & $147(16.1)$ & $86(17.8)$ & $462(18.1)$ \\
\hline More poor & $155(13.4)$ & $154(16.9)$ & $64(13.2)$ & $373(14.6)$ \\
\hline Most poor & $140(12.1)$ & $108(11.8)$ & $65(13.4)$ & $313(12.2)$ \\
\hline Missing & $17(1.5)$ & 17 (1.9) & $3(0.6)$ & $37(1.4)$ \\
\hline
\end{tabular}

${ }^{1}$ NHIS: National Health Insurance Scheme; ${ }^{2}$ HH SES: household socioeconomic status.

TABLE 2: Various types of hypertension prevalence by age group within the surveyed population.

\begin{tabular}{|c|c|c|c|c|}
\hline \multirow[b]{2}{*}{ Raised blood pressure type } & \multicolumn{3}{|c|}{ Respondent's age group } & \multirow[b]{2}{*}{$\begin{array}{l}\text { Total }(2,555) \\
\%(95 \% \mathrm{CI})\end{array}$} \\
\hline & $\begin{array}{c}18-39 \text { years } \\
(N=1,158) \\
\%(95 \% \mathrm{CI})\end{array}$ & $\begin{array}{c}40-59 \text { years } \\
(N=913) \\
\%(95 \% \mathrm{CI})\end{array}$ & $\begin{array}{c}60 \text { and above years } \\
(N=484) \\
\%(95 \% \mathrm{CI})\end{array}$ & \\
\hline Prehypertension & $38.6(35.8-41.1)$ & $44.5(41.3-47.7)$ & $48.3(43.9-52.8)$ & $42.5(40.6-44.5)$ \\
\hline Systolic hypertension & $3.4(2.5-4.6)$ & $8.2(6.6-10.2)$ & $21.5(18.0-25.4)$ & $8.5(7.5-9.7)$ \\
\hline Diastolic hypertension & $3.0(2.2-4.2)$ & $3.9(2.8-5.4)$ & $2.3(1.3-4.1)$ & $3.2(2.6-4.0)$ \\
\hline Hypertension (stage 1) & $8.3(6.8-10.0)$ & $18.8(16.4-21.5)$ & $27.9(24.1-32.1)$ & $15.8(14.4-17.2)$ \\
\hline Hypertension (stage 2) & $2.3(1.6-3.4)$ & $12.0(10.1-14.3)$ & $19.0(15.7-22.7)$ & $9.0(7.9-10.1)$ \\
\hline
\end{tabular}

Prevalence of the different stages of hypertension in male and female subjects is presented in Table 3. Male subjects had a significantly higher prevalence of prehypertension $(p<0.001)$ and stage- 1 hypertension $(p=0.002)$ compared with female subjects.

3.2. Awareness, Treatment, and Control of Hypertension. Out of the 717 respondents who were classified as hypertensive, $45.9 \%$ (95\% CI: 42.2-49.6; 329/717) had in the past been diagnosed and informed by a health practitioner and were aware of their status (Table 4). Level of awareness increased significantly with age group $(p<0.001)$, being highest in the $\geq 60$ years group and lowest in the $18-39$ years group. Out of the 329 hypertensive respondents who were aware of their status, $54.1 \%, 3.6 \%$, and $2.4 \%$ of them had sought for medical, herbal, and both medical and herbal treatments, respectively. Among those who sought medical treatment, $41 \%$ had their raised blood pressure controlled, while 59\% had uncontrolled blood pressure. A higher
TABle 3: Prevalence of the different stages of hypertension among male and female subjects in the study area.

\begin{tabular}{lcccc}
\hline Stage of hypertension & Male & Female & Difference & $P$ value \\
\hline Prehypertension & 49.3 & 38.1 & 11.3 & $<0.001$ \\
Systolic hypertension & 9.3 & 8.0 & 1.3 & 0.252 \\
Diastolic hypertension & 3.3 & 3.2 & 0.1 & 0.887 \\
Stage 1 hypertension & 18.5 & 14.0 & 4.6 & 0.002 \\
Stage 2 hypertension & 8.9 & 9.0 & -0.1 & 0.951 \\
\hline
\end{tabular}

proportion of participants aged $\geq 60$ years had sought treatment (Table 4).

3.3. Risk Factors for Hypertension. Current usage of tobacco was highest (3.2\%) among the age group of 40-59 years' compared with the other age categories (Supplementary 1). Hypertensive respondents who have ever or within the past 12 months or within the past 30 days consumed alcohol were 
TABLE 4: Awareness, treatment, and control of hypertension among the study population.

\begin{tabular}{lcccrr}
\hline & $\begin{array}{c}\text { HPT } \\
(N)\end{array}$ & Aware & $\begin{array}{c}\text { Aware, } \\
\text { (95\% CI })\end{array}$ & $\begin{array}{c}\text { Treated and controlled, } \\
\%(95 \% \mathrm{CI})\end{array}$ & $\begin{array}{c}\text { Aware, not treated } \\
\%(95 \% \mathrm{CI})\end{array}$ \\
\hline Age group (years) & & & & & \\
$18-39$ & 150 & 54 & $36.0(28.7-44.0)$ & $18.5(10.2-31.3)$ & $81.4(68.7-89.8)$ \\
$40-59$ & 309 & 148 & $47.9(42.4-53.5)$ & $37.2(29.7-45.3)$ & $62.8(54.7-70.3)$ \\
$\geq 60$ & 258 & 127 & $49.2(43.1-55.3)$ & $55.9(47.1-64.3)$ & $44.1(35.6-52.9)$ \\
All & 717 & 329 & $45.9(42.2-49.6)$ & $41.3(36.1-46.8)$ & $58.7(53.2-63.9)$ \\
\hline
\end{tabular}

approximately 38\%, 22\%, and 19\%, respectively. Consumption of alcohol within the past 30 days was approximately $18 \%$ for each of the age groups (Supplementary 1 ).

Approximately $85 \%$ and $21 \%$ of hypertensive respondents were involved in active work and sports, respectively. Approximately $10 \%$ of these respondents also had diabetes, with the prevalence being highest $(11.0 \%)$ for respondents within the 40-59 years group. About half of the hypertensive respondents had a normal BMI. Majority of hypertensive respondents had hypercholesterolemia, with proportions being highest (59.9\%) for respondents of $\geq 60$ years. Proteinuria $(\geq 0.3 \mathrm{~g} / \mathrm{L})$ was present in $3.5 \%(25 / 714)$ of hypertensive patients (Supplementary 1).

Risk factors found to be associated with one being hypertensive were current tobacco usage $(p=0.053)$, past tobacco usage $(p<0.001)$, prediabetes $(p=0.042)$, high BMI $(p<0.001)$, hyperglycaemia $(p=0.038)$, and hypercholesterolaemia (0.010), whereas being active in sports and doing vigorous work were both less associated with hypertension $(p<0.001)$ (Supplementary 1$)$. The risk factors were the same for both male and female subjects across the different age groups.

\section{Discussion}

This community-based epidemiological screening study establishes that the prevalence of hypertension is higher than expected in predominantly rural communities in Ghana. The overall prevalence was consistent with what was reported by Bosu as the prevalence of hypertension among adults in Ghana [9], Mohammed et al. among Kenyans [7], and Arrey et al. among Cameroonians [13]. Our value, however, was lower compared with those from other cross-sectional studies in Cameroon [14, 15], Ghana [16], Kenya [17], South Africa [16, 18], and Tanzania [19], where the overall prevalence of hypertension was more than $40 \%$ of the study populations. The high prevalence of hypertension in this rural setting could be attributed to the changing lifestyles of rural residents to increasingly reflect those of urban dwellers, including alcohol abuse, smoking, lack of physical exercise, and high intake of salt and fatty foods. The increased prevalence of hypertension with age reported in this study has been well documented in other studies [1, 20-22]. Arterial stiffening as a result of aging has been described as a contributory factor for the higher predisposition to hypertension among the elderly [23-25].

Our demonstration that more than half of participants with hypertension were not aware of their condition is in agreement with findings in several other studies $[4,7,26]$. Low access to healthcare services and poor health seeking behavior could be contributing factors to the low levels of awareness. The high proportion of persons identified with hypertension and not on treatment reported in our study is similar to the $59.5 \%$ reported by Sanuade et al. [8] in a national survey in Ghana but lower compared with the $84.4 \%$ reported by Mohammed et al. [7] from a national survey in Kenya.

Our findings on the proportion of controlled hypertension in the different age groups were similar to the United States National Health and Nutrition Examination Surveys (NHANES) 1999-2014, which also showed lowest level of control among persons 18-39 years [21]. The nearly hypertension control rate reported in our study was similar to the $51 \%$ reported by Mohamed et al. in Kenya [7] but higher than the nearly one-third prevalence of controlled hypertension in the study by Khader et al. in Jordan [27] and the $23.8 \%$ reported by Sanuade et al. in a national survey in Ghana [8]. Although not optimal, the higher level of hypertension control compared with others could be due to higher levels of compliance to treatment regimens by persons with hypertension in our study area.

The high proportion of persons who knew they were hypertensive but were not on any treatment could be due to poor adherence and subsequent default to treatment. This has been attributed to factors such as inadequate counseling, misconceptions that hypertension is curable, and high cost of drugs [28-30]. More education on hypertension prevention and treatment through task shifting at the community level, taking hypertension (and other NCDs) healthcare to the doorsteps of community members, financing, and availability of hypertension medication through the National Health Insurance Scheme (NHIS) will contribute significantly to improvements in treatment rates among hypertensive individuals who are aware of their status [31]. Factors associated with hypertension in our study area were similar to findings from other studies $[32,33]$. Health education and other interventions should target these risk factors to improve hypertension prevention and management.

\section{Limitations}

A key limitation is that some responses were subjective and self-reported. These responses could have been affected by recall bias. However, the use of well-trained interviewing staff and Show Cards to explain the questions ensured that, as much as possible, accurate responses were obtained. 


\section{Conclusions}

Our study showed that close to one-quarter of adults in the middle belt of Ghana had hypertension with less than half of them being aware of their hypertensive status, and nearly half of those on treatment having controlled hypertension. Healthcare systems need adequate resources that will enable them to screen, educate, and refer identified hypertensive individuals for appropriate management to prevent or minimize the development of complications.

\section{Data Availability}

The data used to support the findings of this study are available from the corresponding author upon reasonable request.

\section{Conflicts of Interest}

All authors declare no conflicts of interest.

\section{Acknowledgments}

The authors are grateful to the community members of the Kintampo North Municipality and Kintampo South District for volunteering to participate in this study and their community leaders, as well as the Directors of Health Services who granted permission for the study, the Ghana Health Service, staff of the Kintampo Health Research Centre who supported the field work, laboratory analysis, and data management. The authors are extremely grateful to the management of KHRC for wholly funding this study. This study was funded wholly by the management of the Kintampo Health Research Centre (KHRC).

\section{Supplementary Materials}

Supplementary 1: the relationship between respondents being hypertensive and various risk factors. (Supplementary Materials)

\section{References}

[1] J. Kishore, N. Gupta, C. Kohli, and N. Kumar, "Prevalence of hypertension and determination of its risk factors in rural Delhi," International Journal of Hypertension, vol. 2016, p. 6, 2016.

[2] D. Lloyd-Jones, R. J. Adams, T. M. Brown et al., "Heart disease and stroke statistics-2010 update," Circulation, vol. 121, no. 7, pp. e46-e215, 2010.

[3] World Health Organization, World Health Statistics 2017: Monitoring Health for the SDGs, World Health Organization, Geneva, Switzerland, 2017.

[4] F. Ataklte, S. Erqou, S. Kaptoge, B. Taye, J. B. EchouffoTcheugui, and A. P. Kengne, "Burden of undiagnosed hypertension in sub-saharan Africa," Hypertension, vol. 65, no. 2, pp. 291-298, 2015.

[5] K. Sawicka, M. Szczyrek, I. Jastrzebska, M. Prasal, A. Zwolak, and J. Daniluk, "Hypertension-the silent killer," Journal of Pre-Clinical and Clinical Research, vol. 5, no. 2, pp. 43-46, 2011.
[6] M. E. Lacruz, A. Kluttig, S. Hartwig et al., "Prevalence and incidence of hypertension in the general adult population: results of the CARLA-cohort study," Medicine, vol. 94, no. 22, p. e952, 2015.

[7] S. F. Mohamed, M. K. Mutua, R. Wamai et al., "Prevalence, awareness, treatment and control of hypertension and their determinants: results from a national survey in Kenya," $B M C$ Public Health, vol. 18, no. 3, p. 1219, 2018.

[8] O. A. Sanuade, S. Boatemaa, and M. K. Kushitor, "Hypertension prevalence, awareness, treatment and control in Ghanaian population: evidence from the Ghana demographic and health survey," PloS One, vol. 13, no. 11, Article ID e0205985, 2018.

[9] W. K. Bosu, "Epidemic of hypertension in Ghana: a systematic review," BMC Public Health, vol. 10, no. 1, p. 418, 2010.

[10] S. Owusu-Agyei, O E A. Nettey, C. Zandoh et al., "Demographic patterns and trends in Central Ghana: baseline indicators from the Kintampo health and demographic surveillance system," Global Health Action, vol. 5, no. 1, p. 19033, 2012.

[11] World Health Organization, WHO STEPwise Approach to Chronic Disease Risk Factor Surveillance-Instrument V2. 1, World Health Organization, Geneva, Switzerland, 2008.

[12] National Heart, Lung and Blood Institute, The Seventh Report of the Joint National Committee on Prevention, Detection, Evaluation, and Treatment of High Blood Pressure (JNC 7), National Heart, Lung and Blood Institute, Bethesda, MD, USA, 2003.

[13] W. T. Arrey, C. A. Dimala, J. Atashili, J. Mbuagbaw, and G. L. Monekosso, "Hypertension, an emerging problem in rural Cameroon: prevalence, risk factors, and control," International Journal of Hypertension, vol. 2016, p. 6, 2016.

[14] L. Fezeu, A. P. Kengne, B. Balkau, P. K. Awah, and J. C. Mbanya, "Ten-year change in blood pressure levels and prevalence of hypertension in urban and rural Cameroon," Journal of Epidemiology \& Community Health, vol. 64, no. 4, pp. 360-365, 2010.

[15] A. Dzudie, A. P. Kengne, W. F. Muna et al., "Prevalence, awareness, treatment and control of hypertension in a selfselected sub-Saharan African urban population: a cross-sectional study," BMJ Open, vol. 2, no. 4, Article ID e001217, 2012.

[16] S. Basu and C. Millett, "Social epidemiology of hypertension in middle-income countries," Hypertension, vol. 62, no. 1, pp. 18-26, 2013.

[17] W. Mathenge, A. Foster, and H. Kuper, "Urbanization, ethnicity and cardiovascular risk in a population in transition in Nakuru, Kenya: a population-based survey," BMC Public Health, vol. 10, no. 1, p. 569, 2010.

[18] M. J. Maseko, G. R. Norton, O. H. Majane, N. Molebatsi, and A. J. Woodiwiss, "Global cardiovascular risk profiles of untreated hypertensives in an urban, developing community in Africa," Cardiovascular Journal of Africa, vol. 22, no. 5, pp. 261-267, 2011.

[19] M. J. Dewhurst, F. Dewhurst, W. K. Gray, P. Chaote, G. P. Orega, and R. W. Walker, "The high prevalence of hypertension in rural-dwelling Tanzanian older adults and the disparity between detection, treatment and control: a rule of sixths?," Journal of Human Hypertension, vol. 27, no. 6, pp. 374-380, 2013.

[20] R. S. Vasan, A. Beiser, S. Seshadri et al., "Residual lifetime risk for developing hypertension in middle-aged women and men: the Framingham Heart Study," JAMA, vol. 287, no. 8, pp. 1003-1010, 2002. 
[21] S. S. Yoon, C. D. Fryar, and M. D. Carroll, Hypertension Prevalence and Control Among Adults: United States, 20112014, US Department of Health and Human Services, Centers for Disease Control and Prevention, National Center for Health Statistics, Hyattsville, MD, USA, 2015.

[22] J. Addo, L. Smeeth, and D. A. Leon, "Prevalence, detection, management, and control of hypertension in Ghanaian civil servants," Ethnicity \& Disease, vol. 18, no. 4, pp. 505-511, 2008.

[23] M. AlGhatrif, J. B. Strait, C. H. Morrell et al., "Longitudinal trajectories of arterial stiffness and the role of blood pressure," Hypertension, vol. 62, no. 5, pp. 934-941, 2013.

[24] I. Ferreira, R. J. van de Laar, M. H. Prins, J. W. Twisk, and C. D. Stehouwer, "Carotid stiffness in young adults: a lifecourse analysis of its early determinants," Hypertension, vol. 59, no. 1, pp. 54-61, 2012.

[25] Z. Sun, "Aging, arterial stiffness, and hypertension," Hypertension, vol. 65, no. 2, pp. 252-256, 2015.

[26] D. Adeloye, C. Basquill, A. V. Aderemi, J. Y. Thompson, and F. A. Obi, "An estimate of the prevalence of hypertension in Nigeria," Journal of Hypertension, vol. 33, no. 2, pp. 230-242, 2015.

[27] Y. Khader, A. Batieha, H. Jaddou et al., "Hypertension in Jordan: prevalence, awareness, control, and its associated factors," International Journal of Hypertension, vol. 2019, p. 8, 2019.

[28] J. Spencer, E. Phillips, and G. Ogedegbe, "Knowledge, attitudes, beliefs, and blood pressure control in a communitybased sample in Ghana," Ethnicity and Disease, vol. 15, no. 4, p. 748, 2005.

[29] K. O. Buabeng, L. Matowe, and J. Plange-Rhule, "Unaffordable drug prices: the major cause of non-compliance with hypertension medication in Ghana," Journal of Pharmacy \& Pharmaceutical Sciences, vol. 7, no. 3, pp. 350-352, 2004.

[30] T. H. Harries, V. Twumasi-Abosi, J. Plange-Rhule, and F. P. Cappuccio, "Hypertension management in Kumasi: barriers and prejudice?," Journal of Human Hypertension, vol. 19, no. 12, pp. 975-977, 2005.

[31] A. de Graft-Aikins, M. Kushitor, K. Koram, S. Gyamfi, and G. Ogedegbe, "Chronic non-communicable diseases and the challenge of universal health coverage: insights from community-based cardiovascular disease research in urban poor communities in Accra, Ghana," BMC Public Health, vol. 14, no. 2, p. S3, 2014.

[32] R. Ibekwe, "Modifiable risk factors of hypertension and sociodemographic profile in Oghara, Delta State; prevalence and correlates," Annals of Medical and Health Sciences Research, vol. 5, no. 1, pp. 71-77, 2015.

[33] J. P. Forman, M. J. Stampfer, and G. C. Curhan, "Diet and lifestyle risk factors associated with incident hypertension in women," Jama, vol. 302, no. 4, pp. 401-411, 2009. 


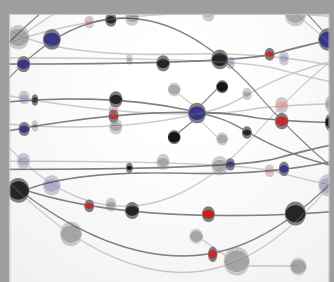

The Scientific World Journal
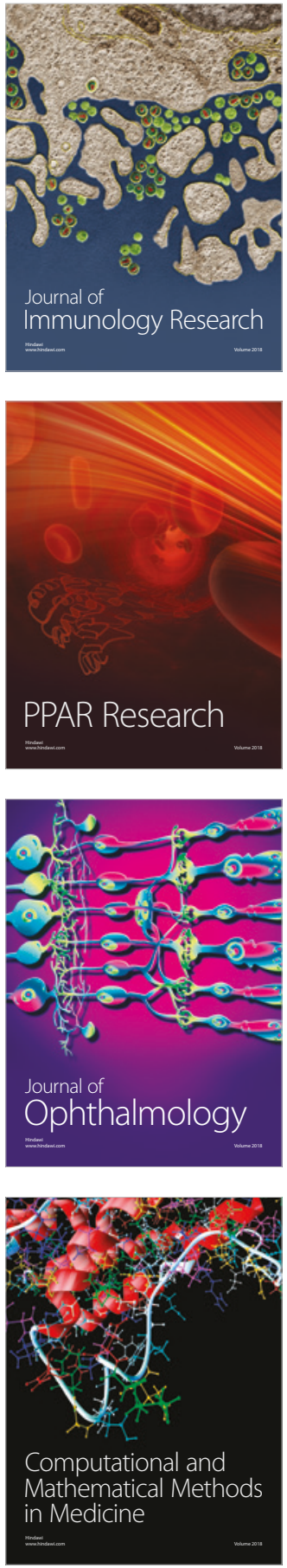

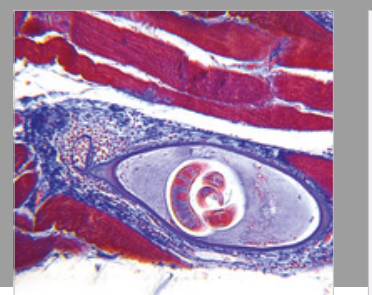

Gastroenterology Research and Practice

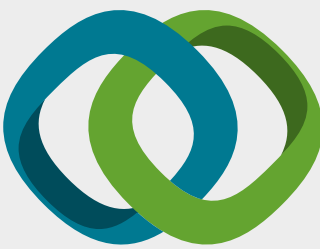

\section{Hindawi}

Submit your manuscripts at

www.hindawi.com
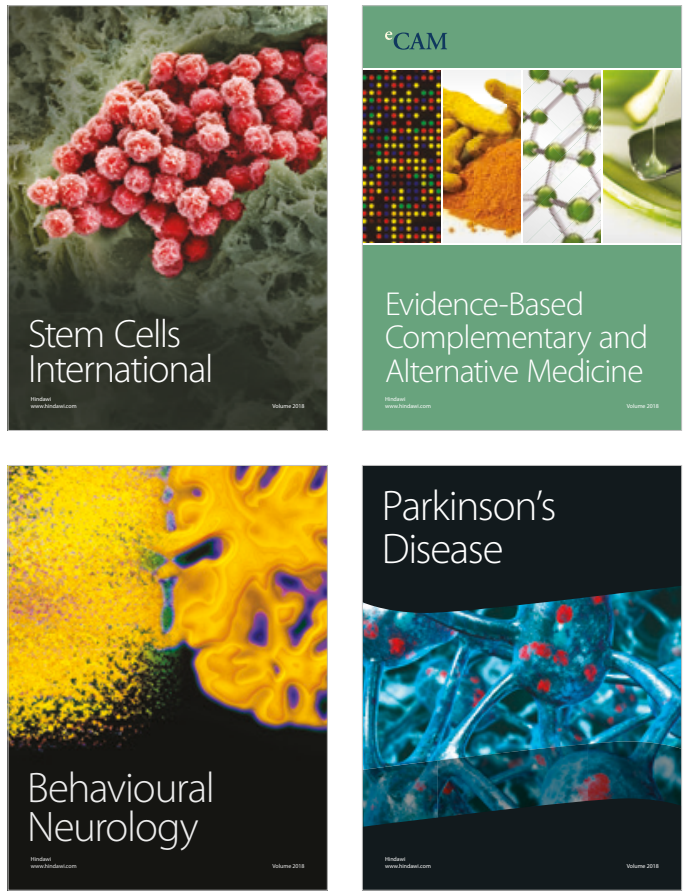

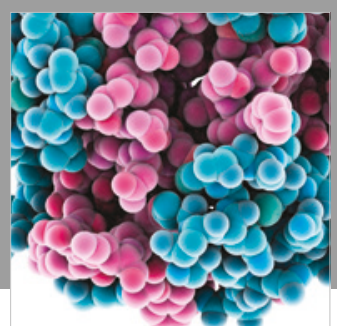

ournal of

Diabetes Research

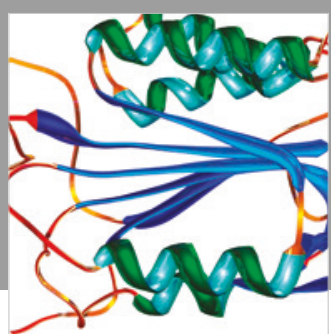

Disease Markers
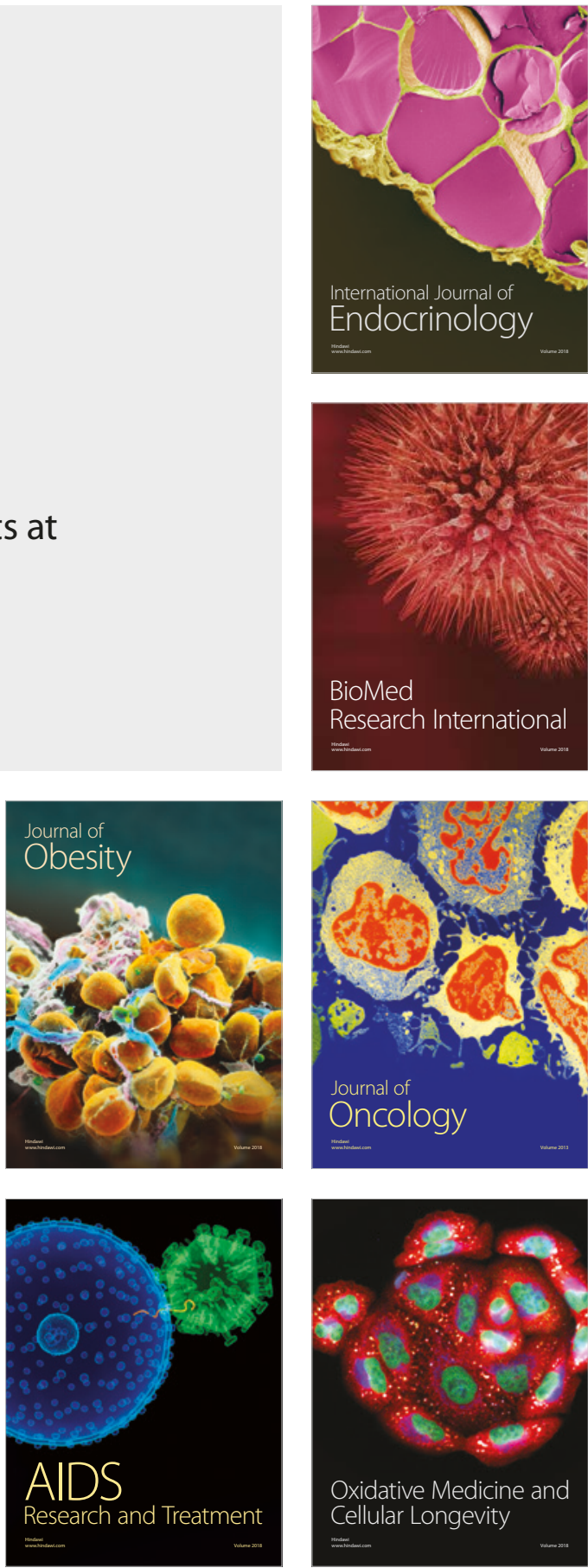